\title{
Session 6: Infectious diseases - II
}

\author{
Thursday 9 October 2003. Moderators: Alois Lang and Katherine Bowdish
}

\section{[11.15-11.45] \\ Engineered recombinant human monoclonal Fab fragment for the determination of antiviral immunoglobulin subpopulations against HCV E2 glycoprotein \\ Roberto Burioni, Nicasio Mancini, Antonella Grieco, Mario Perotti, Silvia Carletti, Filippo Canducci, Pietro E. Varaldo and Massimo Clementi \\ Università di Ancona and Università "Vita e Salute" San Raffaele, Milan, Italy}

The tendency of Hepatitis C Virus (HCV) infection toward chronicity with persistent viral replication in spite of a strong antibody response suggests that in the majority of cases the host immune response is unable to effectively tackle the infection. The role of the humoral response against $\mathrm{HCV}$ envelope glycoproteins (E1 and E2) is still unclear, and clinical data on protection and beneficial effects provided by anti-HCV antibodies are conflicting, even if in some controlled study the protective effect of passive immunotherapy was clearly shown.

Recent data obtained by our group by dissecting the human humoral immunoresponse by molecular cloning of the antibody repertoire of a persistently infected patient in phage display combinatorial vectors shed some lights on the role of antibodies in the virus-host interplay, suggesting that a remarkable part of the humoral immunoresponse seems to be not necessarily beneficial to the host providing a molecular basis to the lack of consistent protection provided by the anti-HCV response. A major advance in understanding the role played by antibodies recognizing the different epitopes would be to correlate their presence with viremia levels, disease progression, therapy effectiveness or other clinical data. Unfortunately the major B epitopes recognized by the human immune system and identified by our group are conformational, and can not reproduced by peptide scan for an ELISA quantitation.

In this presentation we describe the development and the validation of a novel strategy for the in vitro quan- titation of human antibody subpopulations recognizing the B epitopes present on the surface of HCV/E2. We developed a genetic labeling strategy of human recombinant Fabs allowing us to design a competition ELISA test. We furthermore validated our quantitation method constructing mocked serum specimens containing known amount of $\mathrm{IgG1}$ antibody recognizing a given epitope and showing a good correlation of the inhibition of the binding of labeled Fabs with the amount of antibody actually contained in the serum. Finally, we defined a new measure for the amount of antibodies against each single HCV/E2 B epitope present in the serum of the patient named Fab Inhibition Titer (FIT) that we applied preliminarily to a set of $\mathrm{HCV}$ positive sera with different viremia level.

In conclusion, the FIT test described in this paper is a fast and high-throughput assay able to measure the amount of antibodies directed against conformational epitopes present on the E2 surface, and it can be adapted to any other viral or bacterial pathogen. In this particular case, the antibody response against E2 glycoprotein, considered the most important viral structure interacting with cellular targets in the initiation of $\mathrm{HCV}$ infection, can now be dissected and studied to a deeper level, obtaining new information on interaction between antibodies and virus.

\section{[11.45-12.15]}

\section{Development and clinical evaluation of human} monoclonal antibodies for treating $\mathrm{HCV}$ infection R. Eren ${ }^{\mathrm{a}}$, E. Ilan ${ }^{\mathrm{a}}$, O. Nussbaum ${ }^{\mathrm{a}}$, L. Neville ${ }^{\mathrm{a}}$, Y. Arazi $^{\mathrm{a}}$, J. Gopher ${ }^{\mathrm{a}}$, G. Kitzis-Livneh ${ }^{\mathrm{a}}$, A. Zauberman $^{\mathrm{a}}$, E. Galun ${ }^{\mathrm{b}}$ and S. Dagan ${ }^{\mathrm{a}}$ ${ }^{a}$ XTL Biopharmaceuticals Ltd, Rehovot, Israel ${ }^{\mathrm{b}}$ Hadassah University Hospital, Jerusalem, Israel

There are urgent needs to develop effective therapies for managing $\mathrm{HCV}$ infection. Antibodies may be useful to prevent infection following liver transplantation or accidental exposure. They could also be used for 
treatment of patients with chronic infection possibly in combination therapies. Human monoclonal antibodies (HMAbs) to the HCV envelope protein (E2) were generated from peripheral $\mathrm{B}$ cells isolated from individuals infected with HCV genotype 1b. The HMAbs were tested in vitro for their binding, affinity and their ability to immunoprecipitate viral particles from sera of patients infected with HCV from different genotypes. HMAbs having high affinities to E2 and broad immunoprecipitation ability were selected and further evaluated in the HCV-Trimera mouse model that was developed to permit practical in vivo screening of anti-HCV agents. They were capable to inhibit HCV infection of human liver fragments as measured by reductions in mean viral load and in the percentage of HCV positive mice. Their possible role in treatment was demonstrated by their ability to significantly reduce mean viral load when administered to HCV-Trimera mice with established viremia. A HMAb, HCV-AB68, was chosen as a clinical candidate. A phase IA clinical study was designed to test safety, tolerability and efficacy of a single infusion of HCV-AB68 in 15 chronic HCV patients. HCV-AB68 was safe and well tolerated. Significant reduction in $\mathrm{HCV}$ viral RNA levels, ranging from 2 to 100 fold, was demonstrated in 8 out of 15 patients following HCV-AB68 administration. A phase I/II multiple infusions, dose escalating clinical study is currently underway.

\section{[12.15-12.35]}

Prevalence of Hepatitis-C Virus and Hepatitis-B Virus Antibodies in HIV-1 Positive Antenatal Women in Benin City, Nigeria

Anukam Kingsley Chidozie ${ }^{\mathrm{a}}$ and Francis Oronsaye E. ${ }^{b}$

a Department of Pharmaceutical Microbiology, Faculty of Pharmacy, University of Benin, Edo State, Nigeria

${ }^{\mathrm{b}}$ Department of Medical Microbiology, College of Medicine, University of Benin, Edo State, Nigeria

There is a preponderance of agreement that HIV is assuming an unprecedented proportion especially in the developing world. In recent times, screening of antenatal women for HIV antibodies have become a routine in our clinical settings.

However, because of scarce resources, few of our hospitals and clinics have incorporated Hepatitis B and $\mathrm{C}$ virus with HIV screening.

In this study we investigated the sero-prevalence of both hepatitis B and hepatitis C virus in HIV-1 positive antenatal women. Sera were tested using a qualitative immunochromatographic assay for the detection of hepatitis B surface antigen and hepatitis $C$ virus antigen. The method employs a unique combination of anti-HBsAg antibody-dye conjugate (colloidal gold) and a solid phase antibodies to selectively identify surface antigens of hepatitis $B$ virus and hepatitis $C$ virus respectively, with a high degree of sensitivity and specificity.

A total of 1436 pregnant women were tested and 105 (7.3\%) were HIV-1 positive, while 1331 were negative. Of the 105 HIV-1 infected subjects, 36 (34\%) tested positive for anti-HCV, while $42(40 \%)$ tested positive for anti-HBV. $16 \mathrm{HIV}-1$ subjects tested positive for both anti-HCV and anti-HBV representing $15 \%$ of the HIV1 positive subjects.

Despite the reports of anti-HCV loss in HIV-1 infected individuals, routine check of all pregnant women becomes imperative.

Regardless of other treatment considerations, all pregnant women should be vaccinated against hepatitis $\mathrm{B}$ and hepatitis $\mathrm{C}$, as these infections may cause significant morbidity and mortality when superimposed in pre-existing HIV-1 infection.

[13.30-14.15]

[Keynote Lecture]

Recent advances in generation of human monoclonal antibodies

Jim Larrick

Palo Alto Institute of Molecular Medicine, CA, USA

Abstract not received. 